\title{
Aplicação dos modelos matemáticos na distribuição granulométrica da casca de ovo.
}

\author{
Castro, L. S. ${ }^{1 *} ;$ Barañano, A.G. ${ }^{2}$ \\ 1 Programa de Pós-Graduação em Engenharia Química, Universidade Federal do Espírito Santo, Alegre, ES, Brasil. \\ 2 Departamento de Engenharia Rural, Universidade Federal do Espírito Santo, Alegre, ES, Brasil.
}

*e-mail: lucienecasttro@hotmail.com

\begin{abstract}
Resumo
A casca de ovo é um material rico em carbonato de cálcio, porém é um considerado um resíduo para as indústrias de processamento de ovos. Diante da facilidade dessa matéria prima é possível aplicá-lo para aulas práticas de operações unitárias tais como: o peneiramento. $\mathrm{O}$ presente artigo abordou os modelos matemáticos que descreve a distribuição granulométrica de casca de ovo in natura. A partir de um experimento simples será possível explorar diversos conceitos sobre peneiramento. Desse modo, amplia os conhecimentos dos graduandos em relação a teoria e prática. O modelo matemático GGS apresentou melhor ajuste aos dados experimentais.
\end{abstract}

\begin{abstract}
The eggshell is a material rich in calcium carbonate, however it is considered a waste for the egg processing industries. Given the ease of this raw material it is possible to apply it to practical classes of unitary operations such as: sieving. The present article addressed the mathematical models that describe the granulometric distribution of the egg shell sample in natura. From a simple experiment it will be possible to explore several concepts about sieving. In this way, it broadens the knowledge of undergraduates in relation to theory and practice. The mathematic model GGS showed the best fit to the experimentals data.
\end{abstract}

Keywords (Palavras chaves): eggshell, sieving, mathematic model.

\section{Introdução}

A casca de ovo é um material rico em carbonato de cálcio, sendo que é um resíduo gerado expressivamente pela indústria de processamento de ovos, e ainda não há destinação correta para esse resíduo ${ }^{1}$. A obtenção de $\mathrm{CaCO}_{3}$ a partir da casca de ovo envolve diversas operações unitárias tais como: lavagem, moagem, secagem e o peneiramento. A abrangência da obtenção de $\mathrm{CaCO}_{3}$ é uma alternativa para desenvolvimento de prática inovadora relacionada a operações unitárias. Dentre essas destaca-se o peneiramento.

A utilização de resíduos comumente encontrado nos domicílios é forma de conscientizar os graduandos em relação a reutilização de resíduos invés de descartá-lo em aterro sanitário

O presente artigo abordou os modelos matemáticos clássicos para distribuição granulométrica e identificou o melhor modelo que se ajustam aos dados experimentais de distribuição de tamanhos da casca de ovo.

\section{Materiais e métodos}

As cascas de ovo foram lavadas com água destilada. Após a lavagem, as cascas de ovos foram secas numa estufa à $105^{\circ} \mathrm{C}$ durante $24 \mathrm{~h}^{2}$. Em seguidas, as cascas foram trituradas com um auxílio de almofariz e pistilo até a obtenção de um pó fino. A amostra triturada foi peneirada a seco num agitador de peneiras.

A fração mássica $x_{\mathrm{i}}$ corresponde a massa retida na peneira $(m)$ em relação a massa total $(M)$ conforme a Equação $1^{3}$.

$$
x_{\mathrm{i}}=\frac{m}{M}
$$

O diâmetro das partículas $\left(D_{i}\right)$ é obtido a partir da Equação 2.

$D_{i}=\frac{(-) D_{1}+(+) D_{i}}{2}$ 
Nessa equação a simbologia (-) corresponde a massa que atravessou a peneira enquanto a simbologia $(+)$ corresponde a massa retida na peneira.

O diâmetro de Sauter é calculado pela Equação 3.

$D_{\text {Sauter }}=\frac{1}{\Sigma\left(\frac{X_{1}}{D_{1}}\right)}$

Nessa equação $X_{\mathbb{1}}$ é a fração cumulativa. A distribuição granulométrica pode ser descrita por modelos matemáticos. Nesse trabalho focou nos modelos matemáticos clássicos: Gates - Gaudin-Schukman (GGS), Rosin - Rammler-Bennet (RRB) e Sigmóide que são representado pela Equação 4, 5 e 6 respectivamente ${ }^{4,5}$.

$$
\begin{aligned}
& \mathrm{X}=\left(\frac{\mathrm{D}}{\mathrm{k}}\right)^{\mathrm{m}} \\
& \mathrm{X}=\frac{1}{1+\left(\frac{\mathrm{k}}{\mathrm{D}}\right)^{\mathrm{T}}}
\end{aligned}
$$

Nessa equação $X$ é a fração cumulativa, $D$ é 0 diâmetro de partículas, $k, m, n$ e $D^{i}$ são os parâmetros a serem ajustados aos dados experimentais, que são calculados através da linearização. Após a linearização da equação é dado na equação $7, \quad 8$ e $\quad 9$ respectivamente.

$$
\begin{aligned}
& \ln \mathrm{X}=\mathrm{m} \ln (D)-\mathrm{m} \ln (k) \\
& \ln \left[\ln \left(\frac{1}{1-X}\right)\right]=n \ln (D)-n \ln \left(D^{5}\right) \\
& \ln \left(\frac{1-X}{X}\right)=m \ln (k)-m \ln (D)
\end{aligned}
$$

\section{Resultados e Discussões}

A tabela 1 mostra a análise granulométrica da casca de ovo in natura. A amostra apresentou diâmetro de partículas de menores que $0,80 \mathrm{~mm}$ até maiores que

\begin{tabular}{|c|c|c|c|c|c|c|}
\hline $\begin{array}{c}\text { Peneira } \\
\text { Tyler } \\
\text { (mesh) }\end{array}$ & m (g) & $\mathbf{x}_{\mathrm{i}}(\%)$ & $\mathbf{X}_{\mathbf{i}}(\%)$ & $\begin{array}{c}(-) D \\
(m m)\end{array}$ & $\begin{array}{c}(+) D \\
(\mathrm{~mm})\end{array}$ & $\begin{array}{c}\text { D } \\
(\mathrm{mm})\end{array}$ \\
\hline 16 & 0,96 & 1,00 & 99,00 & - & 0,991 & \\
\hline$-16+20$ & 7,33 & 5,00 & 94,00 & 0,991 & 0,833 & 1,41 \\
\hline$-20+28$ & 39,78 & 26,00 & 68,00 & 0,833 & 0,589 & 1,13 \\
\hline 35 & 50,73 & 33,00 & 35,00 & 0,589 & 0,417 & 0,80 \\
\hline Fundo & 52,84 & 35,00 & 0,00 & fundo & fundo & fundo \\
\hline Total & 151,84 & 100,00 & & & & \\
\hline
\end{tabular}
$1,41 \mathrm{~mm}$, já que ficou massa retida $1 \%$ na peneira 16 mesh. Notou-se que $35 \%$ da massa ensaiada apresentou diâmetro menor que $0,80 \mathrm{~mm}$. O diâmetro de Sauter obtido foi de 0,42 $\mathrm{mm}$.

Tabela 1:. Dados do peneiramento da casca de ovo in natura.

As equações características dos modelos matemáticos e o coeficiente de determinação $\left(R^{2}\right)$ para ajuste da reta aos pontos experimentais estão representadas na tabela 2. O modelo matemático GGS apresentou

\begin{tabular}{|c|c|c|}
\hline $\begin{array}{c}\text { Modelo } \\
\text { matemático }\end{array}$ & Equação & $\mathbf{R}^{2}$ \\
\hline GGS & $\mathrm{X}=\left(\frac{\mathrm{D}}{0,6983}\right)^{1,28689}$ & 0,9947 \\
\hline RRB & $X=1-\exp \left[-\left(\frac{D}{1_{2} 0430}\right]\right.$ & 0,9883 \\
\hline Sigmóide & $\mathrm{X}=\frac{1}{1+\left(\frac{0,7551}{\mathrm{D}}\right)^{41621}}$ & 0,8740 \\
\hline
\end{tabular}
melhor ajuste aos dados experimentais. Assim como Matté, Silva e Sfredo ${ }^{6}$ avaliaram a aplicação do modelo matemático GGS e RRB para distribuição granulométrica da aveia e concluíram que o melhor modelo foi GGS.

\section{Conclusões}

Conclui-se que o modelo matemático GGS é o melhor para a distribuição granulométrica da casca de ovo. Desse modo através de um experimento simples é possível envolver diversos conceitos sobre peneiramento, portanto permitindo um fácil aprendizado para os alunos.

\section{Agradecimentos}

Os autores agradecem a FAPES pelo apoio financeiro.

\section{Referências}

[1] CASTRO, L. S. Produção de biodiesel de óleo de algodão utilizando catalisador heterogêneo sintetizado a bpartir da casca de ovo. 77f. Dissertação (Mestrado em Engenharia Química), Programa de Pós-Graduação em Engenharia Química, Universidade Federal do Espírito Santo, Alegre, 2017.

[2] CHAKRABORTY, R.; BEPARI, S.; BANERJEE, A.. Chem. Eng. J., v. 165, p. 798-805, 2010.

[3] Foust, Alan et al. Princípios de operações. Rio de Janeiro: LTC, 1982.

[4] CREMASCO, M. A. Operações unitárias em sistemas particulados e fluidomecânicos. São Paulo: Blucher, 2012.

[5]MASSARANI, G. Fluidodinâmica em Sistemas Particulados. Rio de Janeiro: e-papers, 2002.

[6] MATTÉ, L. S., SILVA, G S.; SFREDO, M. A. Estudo da aplicação de modelos matemáticos para a distribuição granulométrica de aveia em flocos finos. In: Encontro de Educação, Ciência e Tecnologia do IFRS Câmpus Erechim, 2, 2017, Erechim. Anais eletrônicos...Disponível em: <http://erechim.ifrs.edu.br/site/midias/arquivos/2014101 713118708poster_iii_jepex_estudo_da_aplicacao_de _modelos_matematicos.pdf $>$. Acesso 09 de Agosto de 2017 
